\title{
BMJ Open Amphetamine-type stimulant use and self-harm: protocol for a systematic review of observational studies
}

\author{
Craig Cumming (D) , ${ }^{1}$ Gregory Armstrong (D) ,,3 Rohan Borschmann, ${ }^{2,4}$ \\ James A Foulds, ${ }^{5}$ Giles Newton-Howes, ${ }^{6}$ Rebecca McKetin, ${ }^{7}$ Shannen Vallesi, ${ }^{1}$ \\ David Preen, ${ }^{1}$ Jesse Young (D) ${ }^{1,4}$
}

\begin{abstract}
To cite: Cumming $C$, Armstrong G, Borschmann R, et al. Amphetamine-type stimulant use and self-harm: protocol for a systematic review of observational studies. BMJ Open 2022;12:e057029. doi:10.1136/ bmjopen-2021-057029

- Prepublication history for this paper is available online. To view these files, please visit the journal online (http://dx.doi. org/10.1136/bmjopen-2021057029).
\end{abstract}

Received 06 September 2021 Accepted 09 December 2021

Check for updates

(C) Author(s) (or their employer(s)) 2022. Re-use permitted under CC BY-NC. No commercial re-use. See rights and permissions. Published by BMJ.

For numbered affiliations see end of article.

Correspondence to

Craig Cumming;

Craig.cumming@uwa.edu.au

\section{ABSTRACT}

Introduction Amphetamine type stimulant (ATS) use and self-harm are both major public health concerns globally. Use of ATS is associated with a range of health and social problems, and has been increasing internationally in the last decade. Self-harm and ATS use share a number of underlying risk factors and occur at elevated rates in marginalised groups with high rates of exposure to trauma. The relationship between self-harm and ATS use is likely complex, and the causal pathway may run in either direction. A comprehensive review, synthesis and analysis of the evidence are warranted to investigate this relationship and inform policy and practice.

Methods and analysis We will search the Medline, Embase, Cumulative Index to Nursing and Allied Health Literature, PsycINF0 and Scopus databases for relevant observational studies published in peer-reviewed journals. The initial search was conducted on 5 February 2021, with a final search expected on 1 February 2022. All studies will be independently screened by two reviewers, first on title and abstract, and then on full-text to determine inclusion in the review. We place no restriction on the population that studies investigate, our exposure of interest is both prescription and illicit ATS use, comparators will be those not currently using ATS, and our primary outcome of interest is the prevalence of self-harm. Data will be extracted using a predesigned template, and pooled prevalence and pooled measures of effect for the association between ATS use and self-harm. If sufficient data are available, we will perform multiple meta-analyses to produce pooled measures of effect for each measure of ATS exposure, as well as different population sub-groups. The Methodological Standard for Epidemiological Research scale will be used to assess study quality, and Egger's test and $\mathrm{I}^{2}$ values will be used to assess publication bias and heterogeneity, respectively.

Ethics and dissemination No ethical approval is required for this review. We will only synthesise information from published studies that were conducted with ethical approval, so no individual participant data will be used. We will disseminate our findings via publication in a peerreviewed journal, national and international conference presentations, and presentations to stakeholders in the community.

Trial registration number This study has been registered with the International Prospective Register of Systematic Reviews (PROSPER0; CRD42021226562).
Strengths and limitations of this study

- These are both important public health issues globally, and no systematic reviews have previously been performed to synthesise the literature.

- A comprehensive search strategy of five key medical databases, designed with the input of a university librarian, will be used; this will be supplemented by a hand search of the citation and reference list of all included studies.

- If sufficient data are available, a comprehensive analysis plan will be used to ensure all clinically relevant covariates are examined.

- Studies not published in English will not be included.

- In the event a small number of eligible studies are found, heterogeneity between study populations and study design may prevent meta-analysis or the direct comparison between studies.

\section{INTRODUCTION}

The use of amphetamine type stimulants (ATS) is a major public health concern internationally. Estimates suggest that approximately 27 million people globally used ATS in 2018. ${ }^{1}$ Since 2016, countries such as the USA, UK, Germany, The Netherlands, Norway and New Zealand have seen increases in the prevalence of illicit amphetamine use. ${ }^{1}$ Additionally, wastewater analysis across 140 European cities suggests an upward trend since 2011 in the quantity of amphetamines being consumed. ${ }^{1}$ In Australia, there has been a reduction of methamphetamine use among people aged 14 and older since 2001. ${ }^{1}$ However, wastewater analysis suggests an increase of $37 \%$ in consumption of methamphetamine between 2016 and 2019 ${ }^{1}$; likely indicating increasingly heavy use among those who are using. Evidence from the Association of Southeast Asian Nations shows a 93\% increase in treatment admissions for ATS-related treatment between 2017 and 2019, and an $81 \%$ increase in the number of people committing ATSrelated offences during the same period. ${ }^{2}$ It is 
clear from the evidence that ATS use is a growing issue in many parts of the world.

Multiple health and social problems are associated with ATS use. These include poor mental health (including depression, psychosis and suicidal behaviour), ${ }^{3-5}$ hypertension and cardiovascular problems, ${ }^{6}$ violence perpetration, ${ }^{7}$ and contact with the criminal justice system. ${ }^{89}$ Accordingly, there is emerging evidence of increasing ATS-related hospitalisations and health service use in many parts of the world, ${ }^{810}$ placing excess strain on already overburdened services sectors.

Self-harm is defined as intentional self-poisoning or self-injury, irrespective of type of motive or the extent of suicidal intent ${ }^{11}$ and is a major public health issue internationally. ${ }^{12-15}$ ATS use and self-harm share a number of underlying risk factors such as depression and anxiety, ${ }^{16-21}$ and life stressors and traumatic events such as such as physical, sexual and psychological abuse..$^{22-26}$ Both ATS use and self-harm also occur at higher rates in marginalised groups who have experienced high rates of trauma such as those who have contact with the justice system, people who experience homelessness, ${ }^{27-29}$ and lesbian, gay, bisexual, transgender, queer, intersex plus (LGBTQIA+) groups. ${ }^{30-34}$ It is likely that the relationship between ATS use and self-harm is complex; however, it is likely that depression, anxiety, trauma and stressful life events may act as mediators. Conversely, reverse causation (ie, self-medication with ATS for symptoms associated with self-harm) is also plausible.

To our knowledge, no prior systematic review has synthesised the literature and quantified the association between ATS use and self-harm. Accordingly, we will conduct a systematic review which aims to: (1) synthesise the peer-reviewed evidence on the prevalence of selfharm among people who use ATS and (2) summarise and quantify the association between ATS use and self-harm, by comparing self-harm in populations that do and do not use ATS. We will use our findings to inform policy and practice in alcohol and other drugs treatment settings aimed at preventing self-harm and identify key gaps in the knowledge base to direct future research in this area.

\section{METHODS AND ANALYSIS}

This protocol adheres to the Preferred Reporting Items for Systematic Reviews and Meta-Analysis for Protocols ${ }^{35}$ and has been registered with International Prospective Register of Systematic Reviews (PROSPERO) at the University of York, UK (CRD42021226562).

\section{Patient and public involvement}

This study was designed without patient or public involvement.

\section{Eligibility criteria}

Our eligibility and exclusion criteria for this review are presented in table 1 .
Table 1 Study inclusion and exclusion criteria

\begin{tabular}{ll}
\hline Inclusion criteria & Exclusion criteria \\
\hline Peer reviewed literature & No ascertainment of ATS use \\
Reported in English & No reporting of self-harm \\
\hline $\begin{array}{l}\text { Investigating the use of } \\
\text { ATS as an exposure }\end{array}$ & $\begin{array}{l}\text { Studies of individuals (eg, case } \\
\text { reports, case studies) }\end{array}$ \\
Investigating self-harm as & $\begin{array}{l}\text { Studies which are not } \\
\text { observational (eg, trials) }\end{array}$ \\
an outcome & $\begin{array}{l}\text { Studies which do not apply } \\
\text { person-level analysis (eg, }\end{array}$ \\
participants & $\begin{array}{l}\text { ecological studies, systematic } \\
\text { reviews) }\end{array}$ \\
& $\begin{array}{l}\text { Studies exclusively investigating } \\
\text { self-harming ideation }\end{array}$ \\
Using an observational \\
study design
\end{tabular}

ATS, amphetamine type stimulants.

\section{Participants}

We will place no restriction on the populations that studies identified by our search may include. Studies including individuals of any age, and those focussing on subpopulations who have used ATS such as same-sex attracted and gender diverse people, and those with mental illness, will be included.

\section{Exposure measures}

Our exposure of interest is use of prescription and/or illicit ATS; a list of included ATS is provided in box 1.

Studies that identify ATS exposure from any one of the following measures will be considered for inclusion: self-report, valid screening/diagnostic tools and/or International Classification of Diseases Tenth Revision codes (F15, X61, T43.6) where diagnoses are recorded in administrative data. All available data on ATS exposure in addition to those reporting ATS use disorder will be extracted and analysed for: (1) the prevalence of self-harm among people who use ATS and (2) association between ATS use and self-harm. Where available, we will extract data on patterns of ATS use which may include frequency or quantity of use, age of initiation and route of administration. Subject to having sufficient data available, we will compare self-harm between (1) people with and without ATS use disorder; and (2) people with ATS use disorder and other (non-ATS) substance use disorders.

\section{Outcome measures}

Our primary outcome of interest is the prevalence of selfharm; characterised as intentional self-injury or poisoning irrespective of suicidal intent. ${ }^{11}$ Studies investigating suicide (ie, fatal self-harm) or suicidal ideation only will 


\section{Box 1 Substances included in exposure of interest}

Methamphetamine
Amphetamine
N-Methyl-3,4-methylenedioxymethamphetamine
Methylphenidate
Methcathinone
Fenetylline
Ephedrine
Pseudoephedrine
Bupropion
Dextroamphetamine
Mixed amphetamine salts (eg, Adderall)
Lisdexamfetamine
Phentermine

be excluded. Studies that ascertain self-harm by any of the following measures will be considered for inclusion: clinical assessment, administrative health records and/or self-report.

\section{Study design}

We will include observational studies that report individual-level quantitative data. Accordingly, we will exclude studies with the following designs: case reports and case studies (ie, studies reporting on one individual), case series, trials, observational studies not using individual-level analysis (eg, ecological studies) and prior reviews. We will include cohort, case-control and crosssectional studies pursuant to the Cochrane handbook definition of observational studies. ${ }^{36}$

\section{Search strategy}

We will search the following five key health and medical databases for peer-reviewed literature from their inception until the most recent available literature on the date of performing our final search: Medline, Embase, CINAHL, PsycINFO and Scopus. Our initial search was performed on 5 February 2021, we anticipate performing a final update on 1 February 2022. The search strategy used for Medline (Ovid) is displayed in Box 2.

A librarian from the University of Western Australia was consulted during the development of the search strategy. Once full-text screening is completed, the citation and reference lists of all included studies will be searched for additional studies that meet our inclusion criteria but were not identified by our literature search. No restrictions will be placed on the publication year or location.

\section{Study selection and screening}

The research team will use reference management software EndNote $^{37}$ to import studies identified from each database search. Duplicates will be removed in EndNote and all unique studies will be imported into systematic review software Covidence ${ }^{38}$ for screening. Two authors (CC and SV) will independently screen the title and abstract of all identified studies based on our inclusion and exclusion criteria. Conflicts on the eligibility

\section{Box 2 Search strategy Medline (Ovid)}

1. expExp Amphetamineamphetamine/

2. exp 3,4-methylenedioxyamphetamine/ or exp n-methyl-3,4-methylenedioxyamphetamine/

3. (((ATS or stimulant* or amphetamine* or amfetamine* or stimulant* or meth) adj2 amphetamine) or speed or ice or centramina or desoxynorephedrine or fenamine or mydrial or phenamine or phenopromin or thyramine or MDMA or N-Methyl-3,4methylenedioxymethamphetamine or ecstasy or bupropion).ab,ti.

4. exp Amphetamine-Related Disorders/

5. 1 or 2 or 3 or 4

6. exp Self-Injurious Behavior/

7. ("self harm" or "self injur" or "non suicid*" or "self inflict*" or "self wound" or parasuicid" or "para suicid" or "self poison*" or "self mutilat*" or "self cut*" or "self scratch" or "self burn" or "self immolat*" or "suicide attempt*" or "attempt* suicid" or "self-batter"” or NSSI or DSH or "self destruct*").ab,ti.

8. 6 or 7

9. 5 and 8

of a study will be resolved via discussion between the two screening authors, and where consensus cannot be reached, a third author (JY) will be engaged to achieve consensus. Cohen's kappa statistic ${ }^{39}$ will be used to estimate the inter-rater reliability.

Full-text articles will be screened by two authors, with any conflicts resolved by consensus with a third author. Reasons for exclusion will be recorded. Primary reasons for exclusion will include: (1) incorrect exposure, (2) incorrect outcome, (3) incorrect study design, (4) no usable data and (5) unable to obtain the full article. Where study eligibility is unclear from the published fulltext, original study authors will be contacted for clarification. Where it is determined (based on title, authors, locations and dates) that multiple studies have used the same dataset and report the same measures (ie, prevalence and/or measure of association), the study with the longest follow-up time will be included, and the other studies will be excluded from the review.

\section{Data extraction}

A standardised Excel form will be developed a priori by the research team for the purposes of data extraction. Data extraction will be performed by one member of the research team using the standardised form. A second research team member will check the extracted data to identify and correct any errors, and a third research team member will resolve any conflicts by consensus that may arise. A list of fields to be extracted is provided in table 2.

\section{Risk of bias assessment}

The risk of bias assessment will be performed independently by two authors, with a third author resolving any conflicts that cannot be resolved by consensus. The Methodological Standard for Epidemiological Research (MASTER) scale ${ }^{40}$ will used to assess the risk of bias. The MASTER scale provides a single unified tool to assess the risk of bias across different study design types. This is done 


\begin{tabular}{|c|c|}
\hline Author and year of study & $\begin{array}{l}\text { Exposed/non-exposed } \\
\text { conditions }\end{array}$ \\
\hline Geographic location of study & $\begin{array}{l}\text { Median length of follow-up } \\
\text { time and variance }\end{array}$ \\
\hline Study setting & Loss to follow-up \\
\hline Study design & $\begin{array}{l}\text { Statistical methods used (ie, } \\
\text { controlling for confounders, } \\
\text { examining subgroups and } \\
\text { interactions, accounting for } \\
\text { missing data and loss to } \\
\text { follow-up, sensitivity analyses) }\end{array}$ \\
\hline Participant population/group & How bias was addressed \\
\hline Method of recruitment & Measure of association used \\
\hline Data source(s) & $\begin{array}{l}\text { Participant descriptors (eg, } \\
\text { total number, number in each } \\
\text { condition, sex, mean/median } \\
\text { age, other demographic } \\
\text { characteristics of interest) }\end{array}$ \\
\hline Outcome variables & $\begin{array}{l}\text { Main results (unadjusted/ } \\
\text { adjusted estimates) }\end{array}$ \\
\hline Exposure variables & Subgroup analyses results \\
\hline Predictor variables & Any conflicts declared \\
\hline Potential confounders & Any funding declared \\
\hline
\end{tabular}

by assessing each study for the presence of a number of methodological standards aimed at addressing the risk of bias across six potential bias domains (selection, information, design-related, analytic, confounding, external validity) ${ }^{40}$ Adopting the approach of previous systematic reviews, ${ }^{341}$ we will discuss the possible risk of bias and study quality in text and generate a score summarising each study's quality by using the proportion of safeguards against bias that each study incorporated. This is done by using the top scoring study in the review as a reference and assigning each other study a score relative to the reference study's score. For example, if the reference study has a score of 10 , and another study has a score of 8 , the other study will be assigned a bias safeguard score of $80 \%$. The quality summary scores will be used for sensitivity analyses, but not to weight analyses, as recommended by the Meta-analysis Of Observational Studies in Epidemiology study group. ${ }^{42}$

\section{Statistical analysis}

Data analysis will be led by CC with assistance from coauthors. The characteristics of the included studies will be summarised and described. This summary will include study setting and country, study quality, and measures used in the assessment of ATS use and ascertainment of self-harm.

If there are sufficient studies included in the review, meta-analysis will be conducted to determine the pooled prevalence of self-harm among people who use ATS and a pooled measure of effect for the association between use of ATS and self-harm. If sufficient data are available, we will perform multiple meta-analyses to produce pooled measures of effect for each measure of ATS exposure used in the included studies (eg, any ATS use, frequency of ATS use, ATS use disorder) and selfharm. Where studies provide both adjusted and unadjusted effects measures, we will use the adjusted measure consistent with the Cochrane Handbook. ${ }^{36} \mathrm{I}^{2}$ values will be estimated and used to assess heterogeneity. If there are sufficient included studies, meta-regression will also be conducted to investigate the impact of age and study design on pooled estimates if there are sufficient data to do so. Potential publication bias will be assessed using Egger's test. ${ }^{43}$ The meta-analyses results will be presented in forest plots provided there are at least two studies per analysis with acceptable heterogeneity. ${ }^{36}$ The forest plots will be complemented with a harvest plot if appropriate to do so. ${ }^{44}$ If there are insufficient data to conduct metaanalyses, results will be presented using a harvest plot in order to examine study quality, correlations between study characteristics and estimated effect measures. ${ }^{44}$ Informed by the guidance and principles outlined by the Economic and Social Research Council Methods Program, ${ }^{45}$ the harvest plot will be accompanied by a narrative synthesis of the findings of studies included in the review. All statistical analysis will performed using Stata V.16. ${ }^{46}$

Given the evidence of changes in the prevalence of self-harm between adolescence and adulthood, ${ }^{47} 48$ where there are sufficient data extracted we will conduct subgroup/sensitivity analyses stratifying by either a cut-off of $<18 / \geq 18$ years of age or mean/median age if the data do not permit an age of majority cut-off.

Given the evidence of an association between amphetamine use and mental illness, ${ }^{40}{ }^{50}$ if possible we plan to also stratify our analyses by individuals with and without a diagnosed mental illness. Subgroup analyses will also be used to compare studies in which individuals have and have not reported being diagnosed with a substance use disorder. If data from the included studies permit, analyses of subgroups of studies involving LGBTQIA+ populations, people who are homeless, and people with criminal justice system contact will also be conducted as ATS use prevalence is elevated in these groups. ${ }^{27-29} 5152$

If there are a sufficient number of studies included in the review, ${ }^{53}$ separate analyses will be performed for groups of studies based on: type of ATS used; method of self-harm; length of study follow-up time (dichotomised by less or greater than 1 month); and sex of participants. Additionally, analyses will be performed on a subset of studies investigating ATS types that do not have strong hallucinogenic properties (ie, studies investigating methylenedioxyphenylalkylamines such as N-methyl3,4-methylenedioxymethamphetamine alone would be excluded from these analyses). This will be done to investigate whether any association that may be found between ATS use and self-harm is mediated by psychosis, given the association between psychosis and self-harm. ${ }^{54}$ Finally, if there are sufficient data, we will perform a subgroup analysis of studies that report data on ATS withdrawal and 
self-harm to assess a possible relationship between these two issues.

Sensitivity analyses will be conducted which exclude studies assessed to be low quality based on the quality assessment scores (using a median split) to understand the potential impact study quality and risk of bias may have on outcomes; conceptually consistent with the MASTER scale. ${ }^{40}$

Finally, we will assess the strength of the body of evidence of all included studies using the grading of recommendations, assessment, development and evaluations ${ }^{55}$ methodology.

\section{Data statement}

The data extracted from studies included in this review will be published in the University of Western Australia research repository, accessible via doi: $10.26182 / 2 \mathrm{a} 6 \mathrm{~h}-\mathrm{y} 731$.

\section{ETHICS AND DISSEMINATION \\ Ethics}

This systematic review will synthesise data from existing publications, no participants will be recruited and no new participant information will be collected. No ethics approval was required for this review.

\section{Dissemination}

We believe that this will be the first review to systematically search and synthesis evidence investigating the relationship between ATS use and self-harm. It is likely that a substantial proportion of the increase in health service use related to ATS will be attributable to selfharm. Understanding the magnitude of this effect will assist policy-makers to estimate the impact of populationlevel interventions for stimulant use on health service utilisation related to self-harm. Our findings will likely be of interest to academic researchers, clinicians, health service providers and key agency stakeholders and policymakers, as well as people who use or have used ATS. We will disseminate our findings via peer-reviewed publication in an academic journal, and will present our results at conferences. We will also provide our results to relevant professional organisations and government agencies so that it can be disseminated and translated to their members and staff respectively. As part of the publication process, we will publish a plain language synopsis of our work which will include links to the peer-reviewed publication.

\footnotetext{
Author affiliations

${ }^{1}$ School of Population and Global Health, The University of Western Australia, Perth, Western Australia, Australia

${ }^{2}$ The University of Melbourne, Melbourne, Victoria, Australia

${ }^{3}$ Nossal Institute for Global Health, Melbourne School of Population and Global Health, Carlton, Victoria, Australia

${ }^{4}$ Justice Health Unit, Centre for Health Equity, Melbourne School of Population and Global Health, Carlton, Victoria, Australia

${ }^{5}$ Department of Psychological Medicine, University of Otago Christchurch, Christchurch, Canterbury, New Zealand
}

${ }^{6}$ Department of Psychological Medicine, University of Otago, Dunedin, New Zealand ${ }^{7}$ National Drug and Alcohol Research Centre, University of New South Wales, Sydney, New South Wales, Australia

Twitter Craig Cumming @cummingcraig2, Gregory Armstrong @googarmstrong and Shannen Vallesi @ShannenVallesi

Contributors The review protocol was conceptualised by CC and JY with input from RB, GN-H, GA and JAF. JAF, GN-H and RB provided clinical guidance on the search terms and concepts, with RMK providing specialist methodological guidance on search terms and the data extraction plan. JY, JAF and GN-H contributed to the design of the analysis plan. SV and DP along with all other authors have contributed to and approved the final manuscript.

Funding CC is funded by an Australian Government Research Training Program Scholarship (PhD). GA is supported by an Early Career Fellowship from the National Health and Medical Research Council (GNT1138096). JY receives salary and research support from a National Health and Medical Research Council Investigator Grant (GNT1178027).

Competing interests None declared.

Patient and public involvement Patients and/or the public were not involved in the design, or conduct, or reporting, or dissemination plans of this research.

Patient consent for publication Not applicable.

Provenance and peer review Not commissioned; externally peer reviewed.

Open access This is an open access article distributed in accordance with the Creative Commons Attribution Non Commercial (CC BY-NC 4.0) license, which permits others to distribute, remix, adapt, build upon this work non-commercially, and license their derivative works on different terms, provided the original work is properly cited, appropriate credit is given, any changes made indicated, and the use is non-commercial. See: http://creativecommons.org/licenses/by-nc/4.0/.

\section{ORCID iDs}

Craig Cumming http://orcid.org/0000-0002-2556-3294

Gregory Armstrong http://orcid.org/0000-0002-8073-9213

Jesse Young http://orcid.org/0000-0001-5702-372X

\section{REFERENCES}

1 United Nations Office on Drugs and Crime. World drug report 2020, booklet 2: drug use and health consequences. world drug report. Vienna: UNODC, 2020.

2 Kanato M, Choomwattana C, Sarasiri R. ASEAN drug monitoring report 2019. Bangkok: ASEAN Narcotics Cooperation Center, 2020.

3 McKetin R, Leung J, Stockings E, et al. Mental health outcomes associated with of the use of amphetamines: a systematic review and meta-analysis. EClinicalMedicine 2019;16:81-97.

4 Lecomte T, Paquin K, Mueser KT, et al. Relationships among depression, PTSD, methamphetamine abuse, and psychosis. J Dual Diagn 2013;9:115-22.

5 Marshall BDL, Werb D. Health outcomes associated with methamphetamine use among young people: a systematic review. Addiction 2010;105:991-1002.

6 Panenka WJ, Procyshyn RM, Lecomte T, et al. Methamphetamine use: a comprehensive review of molecular, preclinical and clinical findings. Drug Alcohol Depend 2013;129:167-79.

7 McKetin R, Lubman DI, Najman JM, et al. Does methamphetamine use increase violent behaviour? Evidence from a prospective longitudinal study. Addiction 2014;109:798-806.

8 Degenhardt L, Sara G, McKetin R, et al. Crystalline methamphetamine use and methamphetamine-related harms in Australia. Drug Alcohol Rev 2017;36:160-70.

9 Pietsch J, Paulick T, Schulz K, et al. Escalation of methamphetaminerelated crime and fatalities in the Dresden region, Germany, between 2005 and 2011. Forensic Sci Int 2013;233:51-4.

10 Winkelman TNA, Admon LK, Jennings L, et al. Evaluation of amphetamine-related hospitalizations and associated clinical outcomes and costs in the United States. JAMA Netw Open 2018;1:e183758-e58.

11 Hawton K, Saunders KEA, O'Connor RC. Self-Harm and suicide in adolescents. The Lancet 2012;379:2373-82.

12 McManus S, Gunnell D, Cooper C, et al. Prevalence of non-suicidal self-harm and service contact in England, 2000-14: repeated cross-sectional surveys of the general population. Lancet Psychiatry 2019;6:573-81. 
13 , Mokdad AH, Ballestros K, et al, US Burden of Disease Collaborators. The state of US health, 1990-2016: burden of diseases, injuries, and risk factors among US states. JAMA 2018;319:1444-72.

14 Müller A, Claes L, Smits D, et al. Prevalence and correlates of self-harm in the German general population. PLoS One 2016;11:e0157928.

15 Harrison J, Henley G. Suicide and hospitalised self-harm in Australia: trends and analysis. Injury research and statistics series no 93. Canberra: AlHW, 2014.

16 Richardson E, DePue MK, Therriault DJ, et al. The influence of substance use on engagement in non-suicidal self-injury (NSI) in adults. Subst Use Misuse 2020;55:89-94.

17 Breet E, Bantjes J, Lewis I. Substance use and self-harm: a crosssectional study of the prevalence, correlates and patterns of medical service utilisation among patients admitted to a South African Hospital. BMC Health Serv Res 2018;18:1-10.

18 Zubrick SR, Hafekost J, Johnson SE, et al. The continuity and duration of depression and its relationship to non-suicidal self-harm and suicidal ideation and behavior in adolescents 12-17. J Affect Disord 2017;220:49-56.

19 Moller Cl, Tait RJ, Byrne DG. Deliberate self-harm, substance use, and negative affect in nonclinical samples: a systematic review. Subst Abus 2013;34:188-207.

20 Kokkevi A, Rotsika V, Arapaki A, et al. Adolescents' self-reported suicide attempts, self-harm thoughts and their correlates across 17 European countries. J Child Psychol Psychiatry 2012;53:381-9.

21 Toprak S, Cetin I, Guven T. Self-harm, suicidal ideation and suicide attempts among college students. Psychiatry Res 2011;187:140-4.

22 Cerutti R, Manca M, Presaghi F, et al. Prevalence and clinical correlates of deliberate self-harm among a community sample of Italian adolescents. J Adolesc 2011;34:337-47.

23 lob E, Steptoe A, Fancourt D, Abuse FD. Abuse, self-harm and suicidal ideation in the UK during the COVID-19 pandemic. Br $\mathrm{J}$ Psychiatry 2020;217:543-6.

24 Gómez JM, Becker-Blease K, Freyd JJ. A brief report on predicting self-harm: is it gender or abuse that matters? J Aggress Maltreat Trauma 2015;24:203-14

25 Narvaez JCdeM, Remy L, Bermudez MB, et al. Re-traumatization cycle: sexual abuse, post-traumatic stress disorder and sexual risk behaviors among Club drug users. Subst Use Misuse 2019;54:1499-508.

26 Berg MK, Hobkirk AL, Joska JA, et al. The role of substance use coping in the relation between childhood sexual abuse and depression among methamphetamine users in South Africa. Psychol Trauma 2017;9:493-9.

27 Borschmann R, Young JT, Moran P. Ambulance attendances resulting from self-harm after release from prison: a prospective data linkage study. Soc Psychiatry Psychiatr Epidemiol 2017;52:1295-305.

28 Borschmann R, Thomas E, Moran P. Self-Harm following release from prison: a prospective data linkage study. Australian \& New Zealand Journal of Psychiatry 2017;51:250-9.

29 Fazel S, Baillargeon J. The health of prisoners. The Lancet 2011;377:956-65.

30 James S, Herman J, Rankin S. The report of the 2015 US transgender survey, 2016.

31 McKay T, Lindquist CH, Misra S. Understanding (and acting on) 20 years of research on violence and LGBTQ + communities. Trauma Violence Abuse 2019;20:665-78.

32 Kassing F, Casanova T, Griffin JA, et al. The effects of polyvictimization on mental and physical health outcomes in an LGBTQ sample. J Trauma Stress 2021;34:161-71.

33 RKJ T, O'Hara CA, Koh WL. Social capital and chemsex initiation in young gay, bisexual, and other men who have sex with men: the pink carpet $\mathrm{Y}$ cohort study. Substance Abuse Treatment, Prevention, and Policy 2021;16:1-11.

34 Quarshie EN-B, Waterman MG, House AO. Prevalence of selfharm among lesbian, gay, bisexual, and transgender adolescents: a comparison of personal and social adversity with a heterosexual sample in Ghana. BMC Research Notes 2020;13:1-6.

35 Moher D, Shamseer L, Clarke M. Preferred reporting items for systematic review and meta-analysis protocols (PRISMA-P) 2015 statement. Systematic Reviews 2015;4:1.

36 Higgins JPT, Thomas J, Chander J, eds. Cochrane Handbook for Systematic Reviews of Interventions version 6.2 (updated February 2021). Cochrane, 2021.

37 EndNote [program]. EndNote X9 version. Philadelphia, PA: Clarivate, 2013.

38 Veritas Health Innovation. Covidence systematic review software [program]. Melbourne, Australia.

39 McHugh ML. Interrater reliability: the kappa statistic. Biochemia Medica 2012;22:276-82.

40 Stone JC, Glass K, Clark J. A unified framework for bias assessment in clinical research. International Journal of Evidence-Based Healthcare 2019;17:106-20.

41 Borschmann R, Janca E, Carter A. The health of adolescents in detention: a global scoping review. The Lancet Public Health 2020.

42 Stroup DF, Berlin JA, Morton SC. Meta-Analysis of observational studies in epidemiology: a proposal for reporting. JAMA 2000;283:2008-12.

43 Egger M, Smith GD, Schneider M. Bias in meta-analysis detected by a simple, graphical test. BMJ 1997;315:629-34.

44 Ogilvie D, Fayter D, Petticrew M. The harvest plot: a method for synthesising evidence about the differential effects of interventions. BMC Medical Research Methodology 2008;8:8.

45 Popay J, Roberts H, Sowden A. Guidance on the conduct of narrative synthesis in systematic reviews. A product from the ESRC methods programme Version 2006;1:b92.

46 StataCorp. Stata statistical software: release 16. College Station, TX: StataCorp LLC, 2019.

47 Plener PL, Schumacher TS, Munz LM, et al. The longitudinal course of non-suicidal self-injury and deliberate self-harm: a systematic review of the literature. Borderline Personal Disord Emot Dysregul 2015;2:2.

48 Moran $\mathrm{P}$, Coffey $\mathrm{C}$, Romaniuk $\mathrm{H}$. The natural history of self-harm from adolescence to young adulthood: a population-based cohort study. The Lancet 2012;379:236-43.

49 Voce A, Calabria B, Burns R, et al. A systematic review of the symptom profile and course of methamphetamine-associated psychosis. Subst Use Misuse 2019;54:549-59.

50 Salo R, Flower K, Kielstein A, et al. Psychiatric comorbidity in methamphetamine dependence. Psychiatry Res 2011;186:356-61.

51 Hibbert MP, Porcellato LA, Brett CE, et al. Associations with drug use and sexualised drug use among women who have sex with women (WSW) in the UK: findings from the LGBT sex and lifestyles survey. Int J Drug Policy 2019;74:292-8.

52 Thu Vu NT, Maher L, Zablotska I. Amphetamine-type stimulants and HIV infection among men who have sex with men: implications on HIV research and prevention from a systematic review and metaanalysis. J Int AIDS Soc 2015:18:19273.

53 Baker WL, White CM, Cappelleri JC, et al. Understanding heterogeneity in meta-analysis: the role of meta-regression. Int $J$ Clin Pract 2009;63:1426-34.

54 Challis S, Nielssen O, Harris A, et al. Systematic meta-analysis of the risk factors for deliberate self-harm before and after treatment for first-episode psychosis. Acta Psychiatr Scand 2013;127:442-54

55 Goldet G, Howick J. Understanding GRADE: an introduction. J Evid Based Med 2013;6:50-4. 